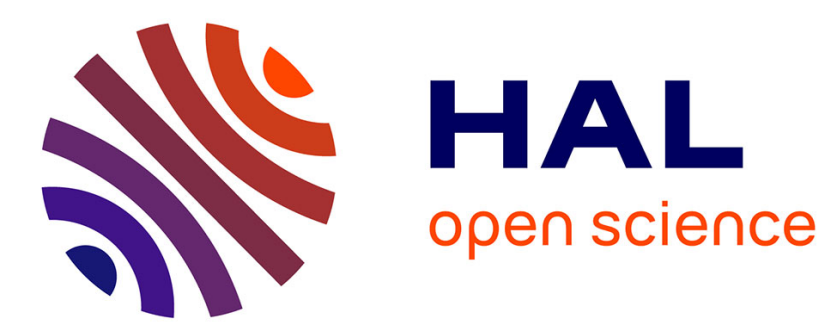

\title{
Evaluation des politiques de transports et équité spatiale
} Alain Bonnafous, Sophie Masson

\section{To cite this version:}

Alain Bonnafous, Sophie Masson. Evaluation des politiques de transports et équité spatiale. Revue d'économie régionale et urbaine, 2003, 4, pp. 547-572. halshs-00068592

\section{HAL Id: halshs-00068592 \\ https://shs.hal.science/halshs-00068592}

Submitted on 13 Mar 2007

HAL is a multi-disciplinary open access archive for the deposit and dissemination of scientific research documents, whether they are published or not. The documents may come from teaching and research institutions in France or abroad, or from public or private research centers.
L'archive ouverte pluridisciplinaire HAL, est destinée au dépôt et à la diffusion de documents scientifiques de niveau recherche, publiés ou non, émanant des établissements d'enseignement et de recherche français ou étrangers, des laboratoires publics ou privés. 


\title{
ASSESSMENT OF TRANSPORT POLICY AND SPATIAL EQUITY
}

\author{
Par \\ Alain BONNAFOUS \\ Professeur \\ et \\ Sophie MASSON \\ Maître de conférences
}

Laboratoire d'Economie des Transports - Université Lyon2-CNRS

ISH, 14 avenue Berthelot

F - 69363 LYON CEDEX 07

Mots-clés : calcul économique, équité spatiale, redistribution, accessibilité, utilitarisme

Key-words : economic assessment, spatial equity, redistribution, accessibility, utilitarianism Classification JEL : D61, D63, R40, R53. 


\section{- I - \\ INTRODUCTION}

Un système de transport irrigue un espace dont tous les lieux ne jouissent pas de la même accessibilité, que cet espace soit local, régional, national ou international. Cela pose le problème de l'inégalité face au droit au transport et plus généralement celui de l'iniquité spatiale. Il est généralement admis, en effet, (et dans de nombreux pays prescrit par la loi) que soit assignée à la politique des transports une pondération d'objectifs. Ces objectifs, parce qu'ils peuvent être contradictoires, forment une problématique dite de mobilité durable qui n'est qu'un aspect particulier de la notion de développement durable qui conjugue les objectifs de croissance, d'environnement et d'équité.

Les économistes abordent, le plus souvent, le problème de l'équité sur le terrain des inégalités sociales ou sur celui, tout proche, de la redistribution des revenus. Ce terrain n'est évidemment pas complètement étranger à la politique des transports (BLOY et alii, 1976). C'est essentiellement d'équité spatiale qu'il sera, ici, question. Elle désigne, elle aussi, une logique de redistribution, mais qui concerne des « avantages » spatiaux. Elle correspond aussi à un principe fondateur de l'aménagement du territoire pour lequel, selon une tradition au demeurant discutable (BONNAFOUS, 1992), la politique du souhaitable ne peut être que celle d'une recherche de l'équité spatiale, consistant à donner des chances comparables de développement à chacun des territoires urbains ou régionaux en résorbant du mieux possible leur déficit d'accessibilité. Les objectifs de la politique de transport intégreraient, alors, un souci d'équité de traitement des individus quelle que soit leur localisation. En somme, un droit au transport égal pour tous.

Observons que le problème de l'équité spatiale, et plus généralement celui de l'aménagement du territoire, ne se pose que si l'on considère que les individus ne sont pas parfaitement mobiles ou que cette parfaite mobilité n'est pas souhaitable. En effet, dans l'hypothèse d'une parfaite mobilité résidentielle des ménages, des individus résidant dans une zone démunie peuvent théoriquement migrer vers une zone dont l'attrait est supérieur. Théoriquement, car dans la réalité, "le degré de mobilité des agents économiques départage, pour l'essentiel, les enjeux entre équité et efficacité » (GUENGANT, 1993).

La prise en compte de l'équité spatiale pose cependant des problèmes. En effet, si le premier objectif de la politique des transports, celui d'allocation optimale des ressources, renvoie à une conception réputée positive - une exigence de recherche d'efficience sous contrainte de rareté, une solution non optimale étant source de gaspillage - l'objectif d'équité spatiale renferme un caractère normatif, dans la mesure où il fait appel à un jugement de valeur pour comparer les différents états économiques pour lesquels efficacité et équité entrent souvent en contradiction. La question qu'il convient de se poser est de savoir comment arbitrer au mieux cette contradiction et, pour cela, comment il convient de la formuler. 


\section{- II - \\ CALCUL ECONOMIQUE ET OBJECTIF D'EQUITE SPATIALE : UNE CONTRADICTION DEMONTRABLE}

Le cadre théorique usuellement retenu pour évaluer les politiques de transport est celui du calcul économique au sens de la «théorie du rendement social » (LESOURNE, 1972).

\subsection{Principe et hypothèses}

Nous savons que ce calcul économique permet une comparaison des états économiques qui requiert l'utilisation d'une fonction d'utilité collective. Son principe est de désigner l'alternative qui maximise la variation de la fonction d'utilité collective ou encore le surplus global de productivité.

Pour rendre opératoire cette valeur très théorique qu'est la variation d'utilité collective et pour la réduire à une sommation algébrique des coûts et des avantages, une hypothèse forte doit être posée: celle de l'indifférence du souverain économique aux bénéficiaires (ou victimes) du passage d’un état économique à un autre. Cela équivaut à supposer que la répartition des revenus est optimale, l'attribution à la marge d'un avantage pouvant alors bénéficier indifféremment à n’importe quel individu. Cette hypothèse, démentie par la société dès lors qu'elle reconnaît la nécessité d'une redistribution, n’est donc qu'une hypothèse de travail nécessaire à l'opérationalité du calcul. La variation d'utilité collective constitue, dans ces conditions, une mesure de l'intérêt collectif du projet évalué, mais une mesure qui évacue toute considération redistributive même si l'hypothèse de répartition optimale peut être levée dans un deuxième temps (BLOY et alii, 1976).

Cette difficulté est aggravée par une contradiction essentielle entre l'objectif d'équité spatiale et cette recherche de l'efficacité du système de transport : les choix d'investissements infrastructurels qui reposent sur ces méthodes tendent à favoriser les liaisons les plus rentables. Or, la rentabilité d'une infrastructure dépend de son utilisation et donc de l'importance des trafics. Ce sont ainsi les corridors les plus denses, ceux qui connaissent des problèmes de congestion, généralement situées dans les régions développées bénéficiant d'un niveau initial d'accessibilité élevé, qui bénéficient de ces investissements.

\subsection{La formalisation de la contradiction}

Pour être bien compris, le conflit peut, en un premier temps, être illustré par deux graphiques simples qui rapportent des variations de surplus à des indicateurs d'accessibilité. L'accessibilité peut être définie comme une mesure locale de la facilité d'accéder, à partir d'une origine donnée, à un ensemble de destination.

Supposons que soit résolue la question d'un zonage pertinent pour l'évaluation des équités ou iniquités territoriales et que, pour l'ensemble des zones concernées, soit établi un indicateur d'accessibilité. Si l'on reporte en ordonnée les avantages nets d'un programme d'investissement ventilés par zone, mesurés par la portion de surplus global de productivité bénéficiant aux habitants ${ }^{1}$ de chaque zone $\left(\Delta S_{\mathrm{i}}\right)$, on peut exprimer le caractère redistributif en reportant en abscisse, pour chaque zone $i$, son indicateur d'accessibilité $\left(A_{i}\right)$. 

propos.

La figure ci-après présente deux configurations fictives permettant d'illustrer notre

Figure 1 - Les effets spatiaux d'un programme d'investissement routier : une relation positive entre rang d'accessibilité des zones et rentabilité globale
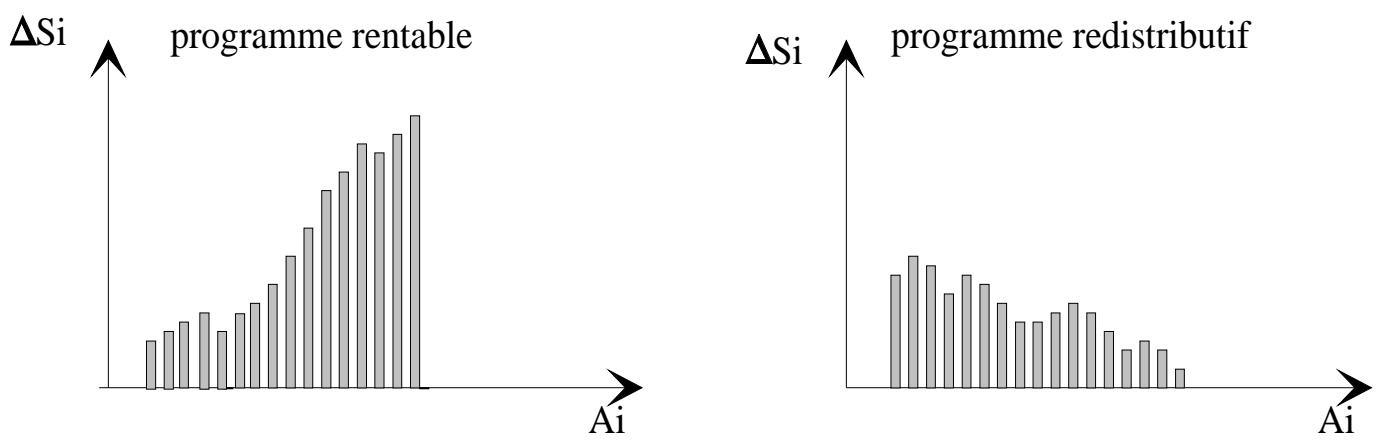

Sur le graphique de gauche, le projet n'est pas redistributif dans la mesure où la variation du surplus est une fonction croissante du rang d'accessibilité, au contraire de ce qui se passe sur le graphique de droite. Nous dirons qu'il y a conflit entre les objectifs de rentabilité et d'équité dès l'instant que les programmes les plus rentables ont généralement une silhouette croissante et que les programmes redistributifs dégagent, a contrario, des surplus de productivité moins importants. Il y a, en matière d'investissement d'infrastructures, une explication forte à ce conflit, qui tient au phénomène de rendements croissants à l'échelle : les investissements les plus rentables concernent les axes lourds et les régions à forte génération de trafic qui jouissent le plus souvent d'un bon niveau d'accessibilité initial.

Cette opposition peut être clairement formalisée en mettant en évidence une relation positive entre le niveau de rentabilité et celui de l'accessibilité (BONNAFOUS, 1992). La démonstration peut être conduite en deux étapes : dans un premier temps il s’agit de montrer la relation positive entre rentabilité et trafic, puis de démontrer la relation croissante entre trafic et accessibilité.

Pour chaque projet ou chaque politique de transport, la relation empirique entre l'indicateur d'accessibilité et le surplus peut être représentée par une droite de régression d'équation ${ }^{2}$ :

$$
\Delta \mathrm{S}_{\mathrm{i}}=\alpha \mathrm{A}_{\mathrm{i}}+\beta+\varepsilon_{\mathrm{i}}
$$

avec $\Delta \mathrm{S}_{\mathrm{i}}$ : variation de surplus liée à un projet pour la zone $\mathrm{i}$,

$A_{i}$ : niveau d'accessibilité de la zone $i$,

$\alpha$ et $\beta$ paramètres de la régression, et $\varepsilon_{\mathrm{i}}$ résidu aléatoire.

Même si cette représentation est grossière, nous poserons par convention que :

- $\quad$ un projet est déséquilibrant si $\alpha>0$,

- un projet est redistributif si $\alpha<0$. 
Avant d'aller plus loin, il convient de rappeler les définitions et les mesures du surplus économique lié à un projet de transport, ainsi que celles de l'accessibilité.

\subsubsection{Le surplus économique lié à un projet de transport}

Nous savons que (LESOURNE, 1972) la variation du surplus global de productivité entre la situation de référence et la situation induite par un projet peut s'écrire :

$$
\Delta \mathrm{S}=\Delta \mathrm{R}+\Delta \mathrm{C}+\sum_{\mathrm{i}} \Delta \mathrm{S}_{\mathrm{i}}
$$

où $\Delta \mathrm{R}$ est la variation de recette du système de transport liée au projet, $\Delta \mathrm{C}$ est la variation du coût du système liée à la réalisation du projet. Le solde de ces deux termes sera d'autant plus élevé que, pour un coût d'infrastructures donné, la recette de celle-ci s’en trouvera accrue. Cela correspond aux vertus bien connues de la massification des flux liée au phénomène de rendement croissant à l'échelle.

La variation de surplus des usagers entre la situation de référence (0) et la situation avec projet (1) s'écrit ${ }^{3}$ :

$$
\Delta \mathrm{S}_{\mathrm{i}}=\sum_{\mathrm{j}} \int_{0}^{1}-\mathrm{T}_{\mathrm{ij}} \mathrm{dc}_{\mathrm{ij}}
$$

où $\mathrm{T}_{\mathrm{ij}}$ est le trafic entre i et $\mathrm{j}$, et $\mathrm{c}_{\mathrm{ij}}$ est le coût généralisé de transport entre $\mathrm{i}$ et $\mathrm{j}$.

La part du surplus de l'usager liée au trafic induit étant généralement de second ordre, on peut simplifier la formule en la négligeant et considérer que :

$$
\Delta \mathrm{S}_{\mathrm{i}} \cong \sum_{\mathrm{i}}-\mathrm{T}_{\mathrm{ij}} \Delta \mathrm{c}_{\mathrm{ij}}
$$

Un projet est donc d'autant plus rentable que les abaissements de coût de transport qu'il entraîne $\left(\Delta c_{i j}\right)$ concernent les liaisons dont les trafics $T_{i j}$ sont importants. Cette règle n’est infirmée que dans le cas où, sur les axes à trafic important, les infrastructures sont déjà fortes et où, pour un niveau donné de financement, les $\mathrm{C}_{\mathrm{ij}}$ ne sont que faiblement abaissés. Cela correspond au principe intuitif selon lequel un pays très richement doté en infrastructures peut orienter ses investissements nouveaux vers les zones à faible trafic et peu équipées. On peut alors faire de l'aménagement du territoire en choisissant tout de même les projets les plus rentables. Une telle situation, que l'on peut appeler situation d'abondance, se rencontre peu dans la mesure où, le plus souvent, les liaisons à fort trafic restent peu ou prou confrontées à des problèmes de congestion de sorte que les investissements en transport y trouvent leur rentabilité. Retenons donc que, hormis la situation d'abondance, les projets d'infrastructures sont d'autant plus rentables qu'ils sont destinés à supporter des flux importants. Cela constitue la présomption $N^{\circ} 1$ (relation rentabilité-trafic).

Nous devons à présent examiner la relation existant entre accessibilité et niveau de trafic. Au préalable, il est nécessaire de rappeler que toute évaluation des politiques de transport se fonde implicitement sur une loi des déplacements. 


\subsubsection{Evaluation des politiques de transport et loi des déplacements}

Les méthodes d'évaluation de la satisfaction de l'usager reposent implicitement sur une loi de distribution des déplacements des usagers (POULIT, 1974). De manière générale, la distribution des déplacements est supposée se réaliser :

1) proportionnellement à un indicateur de masse des zones réceptrices (par exemple, un nombre d'opportunités de déplacement) ;

2) proportionnellement à une fonction rapidement décroissante du coût généralisé de déplacement entre $\mathrm{i}$ et $\mathrm{j}$ (fonction exponentielle décroissante lorsque l'on postule que la variation relative de la demande est proportionnelle à la variation absolue du coût généralisé, ce que nous supposons ci-après).

Cette loi de distribution des déplacements correspond à une logique gravitaire, qui donne lieu à la formulation suivante, dans le cas des déplacements domicile-travail :

$$
\mathrm{T}_{\mathrm{ij}}=\mathrm{k} \times \mathrm{W}_{\mathrm{i}} \times \mathrm{E}_{\mathrm{j}} \times \mathrm{e}^{-\beta \mathrm{cij}}
$$

Avec $W_{i}$, le nombre d'actifs dans la zone $i ; E_{j}$, le nombre d'emplois dans la zone $j$; $k$, un coefficient d’homogénéité ; $c_{i j}$, les coûts généralisés entre i et $\mathrm{j}$; et $\beta$ un facteur de résistance. Ce schéma de déplacements peut évidemment être généralisé à d’autres motifs que le motif domicile - travail.

Cependant, POULIT (1974) souligne que cette formulation caractérise le comportement de l'usager lorsque l'urbanisation est homogène, c'est-à-dire lorsque les caractéristiques démographiques et économiques de chaque zone sont identiques. Comme il n'en est pas ainsi, des "effets de concurrence entre zones " apparaissent, qui peuvent être illustrés de la façon suivante: si une zone d'activité j se trouve à proximité de très importantes zones résidentielles, les emplois qu'elle peut offrir aux actifs de ces zones sont rares. Le choix est donc réduit; il est en tout cas inférieur au choix moyen calculé sur l'ensemble de l'aire agglomérée qui, elle, comporte, à l'approximation près du phénomène de chômage, "autant d'actifs que d'emplois ». En appliquant brutalement la loi générale de distribution des déplacements, on trouverait que le nombre d'actifs qui désirent se rendre en $\mathrm{j}$ en provenance de toutes les zones résidentielles qui entourent cette zone d'activité est supérieur à Ej. Le problème se pose de façon symétrique lorsque la zone d'activité est très étendue et se trouve entourée de zones résidentielles de taille modeste, les actifs désirant se rendre en j seront moins nombreux que les emplois offerts. Cette difficulté, exprimée dans sa forme la plus générale a été résolue par WILSON (1970) qui a introduit des contraintes d'équilibre sur la somme des émissions et des réceptions ainsi que sur une contrainte de coût global de transport et qui a exprimé la forme générale du système de flux qui maximise l'entropie. Nous ne reprenons ici que les équations de cette approche qui nous sont utiles. Les contraintes sont formulées de la façon suivante :

- le nombre total de déplacements effectués à partir d'une zone donnée ne peut excéder le nombre de ses résidents : $\forall \mathrm{i} \in \mathrm{I}, \sum_{\mathrm{j}} \mathrm{T}_{\mathrm{ij}}=\mathrm{O}_{\mathrm{i}}$

- le nombre total de déplacements vers une zone donnée ne peut excéder la capacité d'accueil de cette zone : $\forall \mathrm{j} \in \mathrm{K}, \sum_{\mathrm{i}} \mathrm{T}_{\mathrm{ij}}=\mathrm{D}_{\mathrm{j}}$ 
- enfin, l'ensemble des déplacements doit être réalisé à l'intérieur d'une enveloppe budgétaire globalement donnée : $\sum_{\mathrm{i}} \sum_{\mathrm{j}} \mathrm{c}_{\mathrm{ij}} \mathrm{T}_{\mathrm{ij}}=\mathrm{C}$

$\mathrm{O}_{\mathrm{i}}$ est le nombre de résidents de la zone $\mathrm{i}, \mathrm{D}_{\mathrm{j}}$ est la capacité d’accueil de la zone $\mathrm{j}$. Si l'on considère les déplacements domicile-travail, $\mathrm{O}_{\mathrm{i}}$ correspond au nombre d'actifs de la zone $\mathrm{i}\left(\mathrm{W}_{\mathrm{i}}\right)$ et $\mathrm{D}_{\mathrm{j}}$ correspond au nombre d'emploi de $\mathrm{j}\left(\mathrm{E}_{\mathrm{j}}\right)$.

La spécification du modèle gravitaire, solution du programme de WILSON s’écrit :

$$
\begin{array}{ll}
\mathrm{T}_{\mathrm{ij}}=\mathrm{O}_{\mathrm{i}} \times \mathrm{a}_{\mathrm{i}} \times \mathrm{D}_{\mathrm{j}} \times \mathrm{b}_{\mathrm{j}} \times \mathrm{e}^{-\beta c i j} & \\
\text { avec : } & \mathrm{a}_{\mathrm{i}}=\mathrm{e}^{-\lambda \mathrm{i}} / \mathrm{O}_{\mathrm{i}} \text { et } \mathrm{b}_{\mathrm{j}}=\mathrm{e}^{-\gamma \mathrm{j}} / \mathrm{D}_{\mathrm{j}}
\end{array}
$$

Les conclusions de WILSON reviennent à introduire deux facteurs correctifs $a_{i}$ et $b_{j}$ qui, selon la configuration urbaine, représentent soit des coefficients de rareté, soit des coefficients d'abondance. Ces coefficients permettent de résoudre la triple série d'égalités sur lesquelles repose la distribution des déplacements lorsque les contraintes aux marges interviennent, c'est-à-dire lorsque les émissions et les attractions sont imposées, ce qui revient à considérer comme donné le système de localisation. C'est l'hypothèse que nous retiendrons dans ce qui suit.

La spécification des trafics étant posée, nous pouvons dès lors examiner la relation qui s’établit entre le trafic et l'accessibilité. Une précision sur la définition de l'accessibilité et sur sa mesure est par conséquent nécessaire.

\subsubsection{Un bref rappel sur la définition et la mesure de l'accessibilité}

L’accessibilité a été généralement définie comme la facilité avec laquelle des lieux d'activité peuvent être atteints à partir d'une localisation donnée. Les mesures d'accessibilité sont alors fondées sur le principe que l'espace contraint le nombre d'opportunités accessibles. Au delà de ce point de vue général, les définitions de ce concept diffèrent largement ${ }^{4}$.

Par exemple, si l'accessibilité se mesure par un nombre de destinations pondérées par la fonction de résistance liée au déplacement que chaque destination implique, l'accessibilité Ai relative à la zone i peut s'écrire :

$$
A_{i}=\sum_{j} D_{j} e^{-\beta c_{i j}}
$$

où $\beta$ s'interprète comme la sensibilité du trafic au coût $c_{i j}$.

La confrontation des équations [9], [10] et [11] nous suggère qu'il n’y a pas de lien direct entre les indicateurs d'accessibilité $A_{i}$ et les trafics tels qu'ils s'établissent comme solution au programme de WILSON. Cependant, la formulation adoptée ci-dessus de l'accessibilité est très simplifiée, et est fondée sur l'hypothèse implicite que l'urbanisation est homogène, c'est-à-dire que les caractéristiques démographiques et économiques de chaque zone sont identiques. Lorsque l'urbanisation n'est pas homogène, des effets de concurrence entre zones apparaissent et altèrent cette expression. POULIT (1974) considère que les 
indices d'accessibilité doivent prendre en compte, dans un souci de cohérence, dans le cas général d'urbanisations hétérogènes, les actifs et les emplois pondérés respectivement par les coefficients de rareté ou d'abondance $\mathrm{a}_{\mathrm{i}}$ et $\mathrm{b}_{\mathrm{j}}$ au sens de WILSON. On a ainsi :

$$
A_{i}=\sum_{j=1}^{n} b_{j} E_{j} e^{-\beta c_{i j}}
$$

Par souci de simplification, nous considérons uniquement les déplacements entre le domicile et le lieu de travail sur un système fermé. L'ensemble des déplacements arrivant dans une zone donnée est alors égal au niveau des emplois proposés dans cette zone: $\left(E_{j}=D_{j}\right)$. D'où :

$$
A_{i}=\sum_{j=1}^{n} b_{j} D_{j} e^{-\beta c i j}
$$

En reprenant [9] et [13] on peut écrire l'égalité suivante :

$$
\mathrm{T}_{\mathrm{i}}=\sum_{\mathrm{j}} \mathrm{T}_{\mathrm{ij}}=\mathrm{O}_{\mathrm{i}} \times \mathrm{a}_{\mathrm{i}} \times \mathrm{A}_{\mathrm{i}}
$$

Dès lors, on constate bien une relation de proportionnalité entre les trafics et l'accessibilité.

Signalons qu'une telle relation peut être également démontrée si l'on considère que le modèle gravitaire spécifiant le trafic $T_{i j}$ n'est plus une solution optimisée mais un modèle estimé empiriquement qui restitue une matrice des trafics observés $T_{\mathrm{ij}}$ (BONNAFOUS, 1992) avec :

$$
\mathrm{T}_{\mathrm{ij}}=\mathrm{O}_{\mathrm{i}} \times \mathrm{D}_{\mathrm{j}} \times \mathrm{k} \times \mathrm{e}^{-\beta \mathrm{c}_{\mathrm{ij}}}
$$

Dans cette équation, les $T_{i j}, O_{i}, D_{j}$ et $c_{i j}$ sont observés, les paramètres $\beta$ et $k$ font l'objet d'une estimation. La qualité de l'ajustement, en terme de variance expliquée, valide ou invalide l'hypothèse exprimée par cette équation. Il faut noter que dans cette approche, les variables $O_{i}$ et $D_{j}$ n’ont pas généralement la signification de marge et ne respectent plus les conditions [5] et [6]. Ce sont le plus souvent des indicateurs de «masses » non directement liés aux trafics. Les marges sont, cependant, reconstituées par :

$$
\begin{aligned}
& \mathrm{T}_{\mathrm{i}}=\sum_{\mathrm{j}} \mathrm{T}_{\mathrm{ij}}=\mathrm{kO}_{\mathrm{i}} \sum_{\mathrm{j}} \mathrm{D}_{\mathrm{j}} \mathrm{e}^{-\beta c_{i j}} \\
& \mathrm{~T}_{\mathrm{i}}=\mathrm{k} \times \mathrm{O}_{\mathrm{i}} \times \mathrm{A}_{\mathrm{i}} \\
& \text { avec } \mathrm{A}_{\mathrm{i}}=\sum_{\mathrm{j}=1}^{\mathrm{n}} \mathrm{D}_{\mathrm{j}} \mathrm{e}^{-\beta \mathrm{c}_{\mathrm{ij}}} \text {, formule courante de l'accessibilité. }
\end{aligned}
$$

Nous retrouvons donc dans ces deux approches, l'une plutôt théorique, l'autre pouvant être « calibrée » une relation de proportionnalité entre le trafic et l'accessibilité. Il y a alors corrélation positive entre les trafics et l'accessibilité. Cela constitue la présomption $N^{\circ} 2$.

Ainsi, le fait qu'existe un lien positif entre rentabilité et trafic d'une part, et un lien positif entre trafic et accessibilité d'autre part, nous permet de conclure que les objectifs de rentabilité des investissements et ceux de l'aménagement du territoire sont contradictoires, hors situation d'abondance de l'offre de transport. 
Cette contradiction entre efficacité et équité spatiale étant formalisée, il convient de savoir comment arbitrer entre ces deux objectifs. Le calcul économique répond de façon simple puisqu'il vise à maximiser la rentabilité indépendamment du souci d'équité. Cette limite avait déjà été soulignée auparavant par quelques auteurs, comme BLOY et alii (1976) ou AYDALOT (1985). Ce dernier expliquait que : "l'aménagement du territoire repose sur la certitude que l'application du calcul maximisateur par des agents libres de leurs décisions engendre des effets négatifs qui doivent être corrigés. Or que recherche l'analyse coûtsavantages? Seulement une mesure plus précise du calcul maximisateur. Elle est donc par nature en contradiction avec l'aménagement du territoire et ne peut que servir d'indicateur partiel qui ne saurait suffire ».

\section{- III - \\ DE L'INSUFFISANCE DE LA NOTION DE SURPLUS DES USAGERS A LA THEORIE ECONOMIQUE DE L'ACCESSIBILITE}

Nous explorerons plusieurs pistes qui tentent de lever la contradiction. La première conduit à s'interroger sur celle qui est ouverte par l'intégration de la notion d'accessibilité dans le calcul du surplus de l'usager selon la proposition de KOENIG. Nous verrons alors qu'il convient de raisonner en amont de l'arbitrage entre efficacité et équité, et de poser le problème de la définition de l'équité spatiale et plus fondamentalement des critères d'une redistribution spatiale « juste ». A ce titre, nous verrons de quelle façon les concepts avancés par les théoriciens de la redistribution peuvent être intégrés dans notre analyse.

\subsection{L’introduction de notion d'accessibilité dans le processus d'évaluation : la proposition de KOENIG}

La proposition de KOENIG (1974) est née d'un constat : "Traditionnellement, on mesure l'efficacité d'un système de transport en utilisant des variables familières à l'ingénieur de trafic, telles que temps par déplacement, vitesses moyennes, ou temps total passé par la population en transport. L'idée implicite dans une telle approche est que le service rendu aux usagers par un réseau se mesure au gain global de temps de transport (ou coût généralisé) qu'il procure. [...] Or ce postulat est faux. Il est facile de trouver des cas où le service rendu augmente, bien que le temps global de transport se soit accru ». L'auteur souligne ainsi l'insuffisance de la notion de coût généralisé de déplacement couramment utilisée pour caractériser le point de vue de l'usager. Cette insuffisance est expliquée par le fait que cette option n'intègre en réalité que les seuls facteurs négatifs (coûts monétaires, temps perdus pondérés par les coefficients d'inconfort). Ne prenant en compte qu'un aspect limité du déplacement, la notion du coût généralisé de déplacement ne peut réellement traduire la satisfaction (ou l'insatisfaction) ressentie par l'usager. Car si se déplacer est ressenti par l'usager comme une contrainte, atteindre sa destination est, en revanche, ressenti par lui comme une satisfaction. KOENIG illustre ce phénomène en donnant un exemple. Des études ont mis en évidence les effets de travaux entrepris dans une agglomération pour améliorer la qualité du réseau de transport. L’objectif déclaré étant de réduire les durées et les coûts de transport. Or ce que l'on a constaté, c'est qu'à la mise en service de ces ouvrages, les temps de transport ne variaient presque pas, mais que les longueurs de déplacements, elles, augmentaient. Ce constat s'explique par le fait que l'usager met à profit toute amélioration d'infrastructure pour augmenter la longueur moyenne de ses déplacements plutôt que la réduction de ses temps et ses coûts de transport. Il faut relever que KOENIG oublie que cela 
correspond à un gain de surplus de l'usager qui est bien pris en compte par le calcul économique : le comportement qui consistera à aller plus loin se traduit par un accroissement de la consommation de transport qui vient abonder le gain de surplus. Ainsi, tout indicateur de service se doit d'associer étroitement, aux aspects négatifs d'un déplacement, ses aspects positifs.

L’accessibilité apparaît alors pour certains auteurs (KOENIG, 1974; POULIT, 1974 ; MORRIS, DUMBLE, WIGAN, 1978 ) comme un critère important d'évaluation. L'évaluation des alternatives des plans de transport doit être envisagée en relation avec les activités des agents économiques ou des groupes car les déplacements quotidiens doivent leur existence à la séparation spatiale des activités. Comme l'accessibilité est une fonction à la fois des structures d'occupation des sols et des performances du système de transport, elle est un critère d'évaluation particulièrement bien approprié afin d'évaluer les services fournis par le système de transport aux différentes catégories d'usagers (voir l'espace lui-même).

POULIT (1974) propose l'introduction d'une nouvelle notion : l'indice de choix. Afin de mieux spécifier cette notion, il étudie les conditions à remplir si on fait varier à la fois sur une liaison (i , j) donnée, les conditions d'urbanisation en j (les emplois offerts) et les conditions de transport entre $\mathrm{i}$ et $\mathrm{j}$, pour que la satisfaction de l'usager reste constante. Il parvient à la conclusion qu'il suffit pour atteindre ce résultat, qu'à toute progression linéaire du coût de transport soit associée une progression multiplicative des choix offerts à la destination. C'est donc une fonction logarithmique qui permet de faire correspondre à une progression multiplicative, une progression additive. Il propose alors de considérer le logarithme des biens offerts pour exprimer la satisfaction de l'usager. $\log \mathrm{E}_{\mathrm{j}}$ caractérise (à un facteur près $\lambda$ ) l'intérêt que l'usager attribue à la possibilité de pouvoir disposer à sa destination d'un marché d'emplois, de services ou de biens égal à $E_{\mathrm{j}}$. En retirant de ce terme positif, le facteur négatif, c’est-à-dire le coût généralisé de transport $\left(\mathrm{c}_{\mathrm{ij}}\right)$, il définit la satisfaction ressentie par l'usager qui s'écrit, pour une liaison ij donnée :

$$
\mathrm{S}_{\mathrm{ij}}=\lambda \log \left(\mathrm{E}_{\mathrm{j}} \mathrm{e}^{-\beta \mathrm{cij}}\right)
$$

Cette expression peut être étendue à l'ensemble des liaisons issues de i. Ainsi, les résidents de la zone i, avant d'effectuer leurs déplacements, prennent-ils en compte l'ensemble des destinations potentielles qui leur sont offertes. La satisfaction d'un résident de la zone i vis-à-vis de l'ensemble des biens offerts par l'ensemble des zones j s'écrit alors :

$$
\mathrm{S}_{\mathrm{i}}=\lambda \log \left(\sum_{\mathrm{j}=1}^{\mathrm{n}} \mathrm{E}_{\mathrm{j}} \mathrm{e}^{-\beta \mathrm{cij}}\right) \quad \text { ('note en fin d'article) }
$$

Selon POULIT, cette formulation du «surplus » de l'usager, en intégrant cet indice de choix, possède une force d'interprétation supérieure à celle du type de HOTTELING. Elle permet également de dépasser, dans le cadre de l'évaluation des projets d'investissements de transport, le mythe des effets structurants, c'est-à-dire le principe selon lequel l'arrivée d'une infrastructure de transport, dans une région donnée, crée de la richesse. En somme l'amélioration du système de transport peut permettre aux habitants d'une zone enclavée d'atteindre des destinations jusqu'alors inaccessibles, et donc d'élargir l'univers de choix des individus. Dans ce cas, ce n'est pas tant l'amélioration des conditions de déplacements qui importe que l'élargissement de l'ensemble des choix offerts aux habitants de la zone considérée. Bien sûr, il ne faut pas exclure que cette " ouverture » puisse induire également des effets déséquilibrants. Cet effet de désenclavement peut conduire à un mouvement de concentration, à terme, vers des zones connectées plus développées. 
KOENIG (1974) reprend cette approche en lui donnant une dimension théorique qui s'inscrit dans la tradition néoclassique du comportement du consommateur. Il propose d'intégrer le concept d'accessibilité dans la mesure de l'utilité de l'usager, et définit la notion d'utilité nette. L'accessibilité est alors une notion positive traduisant l'intérêt que l'usager attribue aux choix que lui offre son environnement spatial (urbain, régional, national) desservi par un réseau de transport. L'utilité nette d'un déplacement s'exprime par la différence entre l'intérêt brut (ou utilité brute), qu'il porte au bien ou service qui motive son déplacement à l'endroit où ce bien ou ce service peuvent être obtenus (élément moteur du déplacement), et le coût généralisé du déplacement, qui permet d'accèder à ce lieu (élément résistant). A ce titre, l'accessibilité est un indicateur de la qualité de service offert par l'ensemble d'un réseau de transport, fondé sur ce à quoi il permet d'accéder (les opportunités).

Il convient cependant de formaliser la cohérence entre cette approche de l'utilité (individuelle ou collective) et celle du modèle standard.

\subsection{Les apports de l'association du surplus du consommateur et de l'accessibilité}

NEUBERGER (1971) est le premier à avoir établi l'association entre les indicateurs d'accessibilité de HANSEN et les mesures du surplus des avantages spatiaux du consommateur. Il a calculé le surplus du consommateur, en supposant que les déplacements étaient correctement décrits par un modèle gravitaire à la WILSON. Il trouve que la variation de surplus du consommateur est égale à ${ }^{6}$ :

$$
\Delta \mathrm{S}_{\mathrm{i}}=\text { xo. } \log \mathrm{A}_{\mathrm{i}}
$$

avec xo paramètre de distribution,

$$
\text { et } A_{i}=\sum_{j} O_{j} e^{-\frac{1}{x o} c_{i j}}
$$

Des relations similaires entre surplus du consommateur et accessibilité ont été dérivées par KOENIG sur la base d'une maximisation de l'utilité. Cet auteur a ainsi proposé une théorie économique de l'accessibilité urbaine basée sur les deux hypothèses suivantes :

1) Une hypothèse de comportement du citadin à la recherche d'un emploi : à chaque emploi offert dans l'agglomération, le citadin associe une " utilité nette ", différence entre l'utilité brute de l'emploi (au sens micro-économique c'est-à-dire salaire et intérêt du travail) et sa désutilité brute que constitue le coût généralisé de son déplacement entre son domicile et le lieu de travail. Il choisit alors l'emploi qui lui assure la meilleure utilité nette.

2) L'utilité brute d'un emploi choisi au hasard dans l'agglomération est une variable aléatoire ; la loi de probabilité est supposée être exponentielle négative, ce qui signifie que la probabilité de trouver un salaire (ou utilité nette) supérieur à une valeur donnée décroît très rapidement quand cette valeur augmente. Cela renvoie, en quelque sorte, à l'hypothèse de décroissance de l'utilité marginale. On peut alors démontrer que l'utilité nette moyenne offerte au résident de la zone i s'écrit :

$$
\mathrm{Ui}=\text { xo } \log \mathrm{Ai} \quad \text { avec } \mathrm{Ai}=\sum_{\mathrm{j}} \mathrm{Ej} \mathrm{e}^{-\frac{\mathrm{cij}}{\mathrm{xo}}}
$$

Les usagers se répartissant entre les destinations possibles conformément au principe du modèle gravitaire $1 / \mathrm{x}_{0}$ est ici le coefficient de résistance de ce modèle.

L'intérêt de cette construction théorique est double pour l'auteur: elle permet d'établir une liaison entre le modèle gravitaire classique de distribution du trafic et la théorie 
micro-économique du consommateur ; mais elle permet aussi de procéder à une analyse désagrégée par secteur, ou par catégorie d'usagers de l'utilité d'un projet de transport. Le raisonnement sur l'accessibilité aux emplois se généralise sans difficulté à d'autres opportunités de déplacements (consommation, loisirs, ...).

L'approche en terme d'utilité nette de KOENIG apporte-t-elle une solution au problème de la prise en considération de l'équité spatiale dans les procédures d'évaluation ? Reprenons l'exemple qu'il donne en utilisant un indicateur tel que $A_{i}$ dans une situation où l'on aurait à comparer deux variantes de transport I et II pour deux individus $\mathrm{A}$ et $\mathrm{B}$ avec les valeurs suivantes de l'accessibilité $A_{i}$ (aux emplois par exemple) :

Tableau 1- Comparaison de deux variantes de transport I et II pour deux individus A et B à partir des valeurs de l'accessibilité $\mathrm{A}_{\mathrm{i}}$

\begin{tabular}{|c|c|c|}
\hline variante & $\begin{array}{c}\text { amélioration de la voirie pour } \\
\text { l'automobile } \\
\text { (I) }\end{array}$ & $\begin{array}{c}\text { amélioration des transports } \\
\text { collectifs } \\
\text { (II) }\end{array}$ \\
\hline A (sans voiture) & 1000 & 2000 \\
\hline B (avec voiture) & 22000 & 20000 \\
\hline somme des accessibilités & $1000+22000=23000$ & $2000+20000=22000$ \\
\hline somme des utilités & $\log 1000+\log 22000=16,9$ & $\log 2000+\log 20000=17,5$ \\
\hline
\end{tabular}

Source : KOENIG G. (1979). "A propos de :"Réflexions sur la notion d'accessibilité", Les Cahiers Scientifiques de la revue Transports, $4^{\circ}$ trimestre, pp. 33-40.

On voit que la maximisation des sommes des $A_{i}$ aboutit au choix de la solution routière, alors que la maximisation de la somme des $U_{i}$ aboutit à préférer la solution des transports collectifs. L'utilisation de ce deuxième indicateur suggère donc qu'il vaut mieux augmenter de 1000 unités l'accessibilité $A_{i}$ d'un citadin mal servi ( $A_{i}$ faible) plutôt que d'augmenter de 2000 unités l'accessibilité d'un citadin bien servi ( $A_{i}$ fort). Cela peut être clairement justifié par le fait que la sommation des accessibilités revient implicitement à prendre en considération une fonction d'utilité collective définie par $\mathrm{W}_{1}=\sum_{\mathrm{i}} \mathrm{A}_{\mathrm{i}}$, alors que la sommation des utilités nettes de KOENIG revient à considérer la fonction d'utilité collective : $\mathrm{W}_{2}=\sum_{\mathrm{i}} \mathrm{x}_{0} \log \mathrm{A}_{\mathrm{i}}$. Or $\delta \mathrm{W}_{1} / \delta \mathrm{A}_{\mathrm{i}}=1$, ce qui signifie que toutes les variations marginales d'accessibilité ont une valeur identique, quel que soit leur niveau initial, alors que $\delta \mathrm{W}_{2} / \delta \mathrm{A}_{\mathrm{i}}$ est une fonction décroissante de l'accessibilité de chaque zone, ce qui signifie que la variation d'accessibilité pèse d'autant plus que son niveau est faible. Autrement dit, pour KOENIG (1979), "de tels calculs effectués avec l'indicateur $A_{i}$ sont plus nuisibles à l'équité sociale et l'équité spatiale - qu'un simple calcul d'optimisation du surplus économique, lui-même considéré souvent (et à juste titre) comme insuffisant sur ce point ». Ainsi, il semblerait que l'intégration de l'accessibilité dans la fonction d'utilité permet de mieux prendre en considération des objectifs redistributifs.

Cependant, il convient de se demander sous quelles conditions la proposition de KOENIG fonctionne. Le problème est de savoir si l'utilité nette de KOENIG ne revient pas tout simplement à déplacer les limites du calcul économique sans vraiment s'en affranchir. En effet, gardons à l'esprit les hypothèses de départ : celles redevables au cadre général de la théorie micro-économique (hypothèse de comportement rationnel du consommateur, 
répartition optimale des revenus et recours aux concepts d'utilité individuelle ou collective) ; mais aussi le choix a priori d'une loi de probabilité pour l'utilité brute d'un emploi pris au hasard, qui permet de retrouver la forme souhaitée de l'indicateur d'accessibilité, le modèle de distribution gravitaire du trafic et, enfin, la théorie du surplus. La définition de l'utilité nette de KOENIG permet ainsi d'introduire une pondération par les indicateurs d'accessibilité dans la fonction d'utilité collective et d'exprimer « plus » une logique redistributive.

Mais cela suppose réunies certaines conditions, entres autres celle de la comparabilité des niveaux d'accessibilité entre zones ou régions. Le problème de l'agrégation des indicateurs d'accessibilité, comme le reconnaît d'ailleurs KOENIG (1979), est un problème fondamental. Il est en effet normal de chercher à utiliser les indicateurs d'accessibilité pour comparer diverses situations. Tant que l'on compare diverses situations pour un même individu, le problème posé est relativement simple, même si la solution est moins évidente qu'il n'y paraît. Il s'agit de trouver un indicateur d'évaluation autant que possible exempt de grosses incohérences; des indicateurs tels que $U_{i}$ et $A_{i}$ (avec une fonction décroissante du coût de transport pour la pondération des opportunités) ou même des indicateurs isochrones peuvent fournir une solution acceptable. Tout autre - et beaucoup plus difficile - est le problème de la recherche d'un indicateur d'évaluation agrégé au niveau d'une population ou d'un groupe quelconque de plusieurs individus. Utiliser comme indicateur agrégé l'indicateur d'utilité nette $U_{\mathrm{i}}$ suppose qu'il soit indifférent d'attribuer une unité de surplus économique à un individu ou à un autre indépendamment de son niveau d'accessibilité. KOENIG fait judicieusement remarquer qu'il ne faudrait cependant pas croire que l'utilisation d'un autre indicateur d'accessibilité résoudrait ce problème car l'additivité d'un indicateur quelconque suppose nécessairement qu'il est considéré comme indifférent d'offrir une unité de cet indicateur à un individu ou à un autre.

\section{- IV - \\ DE LA LIMITE DU PRINCIPE DE MAXIMISATION DE L'UTILITE COLLECTIVE DU CALCUL ECONOMIQUE VERS UNE INTEGRATION POSSIBLE DE L'EQUITE}

Dans la première partie, nous avons mis en évidence que les principes du calcul économique tendent à proposer des allocations d'investissements infrastructurels peu compatibles avec un souci d'équité. La solution que propose le calcul économique peut, certes, déboucher sur une solution équitable, mais il s'agit alors d'une coïncidence dans la mesure où le calcul n'intègre pas une norme explicite et autonome de justice. Et comme le souligne THISSE (1994), après beaucoup d'autres, "un conflit subsiste entre équité et utilitarisme ».

Avant d'aller plus loin et d'essayer d'explorer des solutions alternatives au calcul économique, il est indispensable de s'interroger sur ce que l'on entend par une distribution spatiale « juste », d'expliciter, en somme, un principe de l'équité spatiale. Cela pose une question normative : le souci de justice est relatif à la façon dont sont distribués entre les individus les avantages et les charges associés aux différents états de l'économie de manière à ce que chacun ait « son dû ».

Dans un premier temps, nous rappellerons différentes conceptions de l'équité en reprenant les différentes déclinaisons de la fonction de justice sociale proposée par WOLFELSPERGER (1995). Puis, avec la théorie de RAWLS, nous rappellerons de quelle 
façon un concept d'équité peut être formalisé théoriquement. Nous verrons enfin de quelle façon ces différentes approches peuvent aboutir à des propositions complémentaires au calcul économique standard pour l'évaluation des projets de transport, et en exposerons leurs limites.

\subsection{De la recherche d’une conception de l'équité spatiale aux propositions néo- utilitaristes}

\subsubsection{La recherche d'une conception de l'équité spatiale}

Dans l'absolu (un absolu naïf), une situation parfaitement équitable spatialement serait une situation où chaque individu pourrait bénéficier d'une même qualité de service de transport. Cette dernière pouvant être appréhendée par la notion d'accessibilité, la situation parfaitement équitable serait celle où tous les individus supporteraient le même coût généralisé de transport pour satisfaire les mêmes besoins de déplacement. Pourtant, il n’est pas évident que cette solution soit souhaitable, en raison de ses coûts exorbitants pour la collectivité et de la détérioration de situation qu'elle impliquerait pour le plus grand nombre. Il est donc nécessaire de dépasser la conception égalitariste de l’équité.

Un projet équitable pourrait être défini, par exemple, comme visant à minimiser la variance de distribution des accessibilités. Le critère de choix serait alors de minimiser la variance sous contrainte d'efficacité du projet. Mais comme l'observe THISSE (1994), qui étudie ce problème à propos de la localisation d'un service public, il semble que si cette solution peut améliorer les choses en pratique, la question de fond reste la même : quel sens y a-t-il à réduire les disparités entre les zones, si en même temps la situation des zones les moins accessibles se détériore ? Il faut donc rechercher un critère de justice qui permette de prévenir une telle contradiction. Ce critère peut consister à choisir un projet qui minimise le coût d'accès maximal ou a contrario qui maximise l'accessibilité minimale.. Ce principe renvoie à ce que les économistes appellent le principe du maximin et est souvent associé à la théorie de la justice de RAWLS. Nous allons voir de quelle façon ce principe peut être formalisé et être intégré dans une fonction d'utilité collective.

\subsubsection{L'approche néo-utilitariste de l'équité : une intégration possible de l'équité dans la fonction d'utilité collective}

En économie, la recherche dans ce domaine s’est longtemps concentrée sur la question de comparabilité interpersonnelle des valeurs et des bien-être. "Pour pouvoir juger de l'équité de telle ou telle distribution des avantages matériels, n'est-il pas nécessaire de comparer les bien-être de plusieurs individus distincts? Dans ce cas, quels sont les fondements de ces comparaisons interpersonnelles? Le livre de ARROW ("Social choice and individual values », 1963) et son fameux théorème d'impossibilité mettent rigoureusement en évidence cette difficulté: si l'on souhaite agréger tout vecteur de préférences ordinales individuelles en une préférence collective, et cela sans faire appel à la moindre comparabilité interpersonnelle, alors on aboutit inévitablement à identifier le bien-être collectif à celui d'un seul des membres de la société » (PIKETTY, 1994). Cela renvoie au problème, déjà évoqué en première partie, de l'agrégation des préférences individuelles. Le calcul économique suppose alors l'existence d'une autorité supérieure capable de préciser un ordre de préférence entre deux états économiques quelconques et définit une fonction particulière d’utilité collective. 
Nous allons voir que cette fonction d'utilité collective peut prendre différentes formes et intégrer une norme d'équité.

WOLFELSPERGER (1995) propose une conception néo-utilitariste de la justice ${ }^{7}$ «Le caractère « néo » a pour fonction d'attirer l'attention sur le caractère plus général de la construction théorique élaborée sur cette base par rapport à la théorie utilitariste au sens strict». On suppose l'existence d'un observateur idéal, représentant les préférences des individus $^{8}$. La théorie de la justice de type utilitariste peut être synthétisée sous la forme d'une fonction telle qu'à chaque ensemble de valeurs prises par les utilités individuelles corresponde une valeur d'un indicateur qui «mesure» la justice de chacune de ces répartitions. Les économistes néo-utilitaristes définissent une fonction de bien-être social de BERGSON et Samuelson appelée également fonction de justice sociale. Cette fonction est définie par $\mathrm{F}\left(\mathrm{U}_{1}\left(\mathrm{x}_{1}\right), \mathrm{U}_{2}\left(\mathrm{x}_{2}\right), \ldots, \mathrm{U}_{\mathrm{n}}\left(\mathrm{x}_{\mathrm{n}}\right)\right)$ où chaque $\mathrm{U}_{\mathrm{i}}\left(\mathrm{x}_{\mathrm{i}}\right)$ est le niveau d'utilité que la consommation de l'assortiment du vecteur de bien $\mathrm{x}_{\mathrm{i}}$ permet à l'individu i d'atteindre. La fonction de justice sociale peut être, par exemple, définie mathématiquement comme suit ${ }^{9}$ :

$$
\begin{array}{ll}
\mathrm{W}=\frac{1}{\alpha} \sum\left(\mathrm{U}_{\mathrm{i}}\right)^{\alpha} & \text { si } \alpha \neq 0 \\
\mathrm{~W}=\sum \log \mathrm{U}_{\mathrm{i}} & \text { si } \alpha=0
\end{array}
$$

Ainsi, les diverses variantes de la conception néo-utilitariste de la justice peuvent apparaître mathématiquement selon les valeurs attribuées au paramètre $\alpha$ qui sert à pondérer les utilités individuelles dans la définition de la fonction de justice sociale. La valeur prise par le paramètre $\alpha$ traduit de façon numérique le jugement de valeur qui caractérise la sensibilité à l'inégalité et donc la conception de la justice de celui qui s'exprime sur les allocations réalisables.

1) Si $\alpha>1$, on constate que $\delta W / \delta U_{i}$ croît avec l'utilité de chaque individu ce qui correspond à une préférence pour l’inégalité.

2) Si $\alpha=1$, on constate que $\delta W / \delta U_{i}=1$, ce qui signifie que toutes les variations individuelles d'utilité ont une valeur identique, quel que soit le niveau initial d'utilité de l’individu considéré. Nous retrouvons ainsi le principe de l'analyse coûts-avantages.

3) Si $\alpha=0, W=\Sigma \log U_{i}, \delta W / \delta U_{i}$ est une fonction décroissante de l'utilité de chaque individu, ce qui signifie que la variation d'utilité d'un individu compte d'autant plus que son niveau de bien être est faible. Nous retrouvons ici, en quelque sorte, la proposition de KOENIG.

4) Si $-\infty<\alpha<1$, plus $\alpha$ diminue, plus est forte l'aversion pour l'inégalité. Cette aversion croissante pour l'inégalité se traduit graphiquement par des courbes d'indifférences de plus en plus convexes.

5) Si $\alpha$ tend vers moins l'infini, la fonction de justice sociale s'écrit $W=\operatorname{Min}\left(U_{1}\right.$, $\left.\mathrm{U}_{2}, \ldots, \mathrm{U}_{\mathrm{n}}\right)$. La pondération donnée aux utilités est telle que seule compte celle de l'individu dont le niveau de bien-être est le plus faible. Cela définit le principe du maximin, dont nous avons vu qu'il pouvait être une forme pertinente de l'équité spatiale. Nous verrons plus loin qu'en outre ce principe est cohérent avec la théorie de la justice de RAWLS.

Ainsi, il paraît possible d'introduire une certaine répugnance de la collectivité à l’iniquité, et de réconcilier marché et inégalités, par l’intermédiaire de la définition de la fonction de l'utilité collective et l'intégration d'une pondération $\alpha$. 


\subsection{Une tentative d'application au problème de l'évaluation des transports}

\subsubsection{La recherche des fondements théoriques à l'équité : la proposition de RAWLS}

Si la formalisation de la fonction d'utilité collective précédente a le mérité de rendre explicite les différents degrés d'intégration de l'équité en fonction d'un paramètre $\alpha$, on ne peut, cependant, qu'être frappé par la pauvreté du fondement donné à la revendication d'équité et son caractère typiquement subjectif, ce qui réduit considérablement son intérêt pour tous ceux qui ne partagent pas spontanément cette sorte d'intuition éthique et qui souhaitent pouvoir établir leurs discours dans ce domaine sur des bases argumentées. L'approche de RAWLS permet de répondre à cette lacune. WOLFELSPERGER (1995) souligne que le mérite principal de la théorie de la justice de RAWLS qui « est précisément de fournir un fondement philosophique très élaboré au choix de la pondération particulière des utilités qui caractérise la fonction de justice sociale qu'on peut lui faire correspondre ». Cette dernière correspond pour RAWLS à la pondération de $\alpha$ tendant vers l'infini définissant le principe du maximin.

RAWLS $^{10}$ (1971) développe une théorie de la justice en marge de l'approche utilitariste car d'une part, la variable pertinente pour lui n'est pas l'utilité mais un vecteur de biens dits "premiers » que tout individu est censé rechercher (richesse, droits, "sens de sa propre valeur», ...). D’autre part, sa théorie tente de résoudre les «difficultés de comparabilité interpersonnelle, en fondant ses principes sur des actions et des opportunités individuelles dont l'universalité permet de dépasser le problème de l'agrégation des valeurs individuelles, espérant ainsi construire une alternative crédible à l'utilitarisme » (PIKETTY, 1994). RAWLS définit en effet le concept de justice à un niveau philosophique. Ses principes reposent sur la théorie d'un contrat social entre les individus qui se sont réunis pour vivre ensemble. L'individu qui accepte d'être citoyen d'une société ignore cependant quelle sera son incarnation dans la société future, c'est à dire quel citoyen il sera, quelle sera sa position sociale, quelles seront ses propres préférences. Cette absence d'information, que RAWLS appelle le «voile de l'ignorance », correspond à l'impossibilité d'associer des probabilités aux différentes éventualités. L'individu est alors défini comme étant un être rationnel, égoïste ${ }^{11}$ et ayant une forte aversion pour le risque : "Comme la situation d'ignorance dans laquelle ils choisissent de raisonner implique qu'ils ne peuvent savoir quelle position ils occuperont dans la société, ils vont être amenés à s'imaginer dans la position de chacun des membres de la communauté et ainsi, tout en étant égoïstes, à être parfaitement impartiaux et à exprimer des choix comme si leurs préférences tenaient compte de l'intérêt des autres. Les principes de base devant régir la société qui seront choisis unanimement dans cette "position originelle » pourront être déclarés justes » (WOLFELSPERGER, 1995). L’un des principes qui en découle vient que les inégalités sociales et économiques sont justes dans la mesure où elles bénéficient aux membres les plus défavorisés de la société. Ce principe renvoie au principe du maximin des économistes et résulte du voile de l'ignorance. Par exemple, un individu qui doit choisir la répartition des revenus entre 100 centiles va préférer la distribution des revenus qui maximise le revenu du premier centile (le plus pauvre) sachant qu'il a une chance de s’y trouver dans la réalité. Le principe de différence de RAWLS ne 
repose ni sur la bienveillance, ni d'ailleurs sur la malveillance. Il ne conduit pas non plus à choisir la distribution des revenus la moins inégale : en effet, tout accroissement du taux de concentration qui correspond à une augmentation du revenu du plus pauvre est, pour RAWLS, souhaitable.

Ce critère appliqué au domaine des transports revient à considérer que les inégalités dans les qualités de desserte qui sont jugées acceptables sont celles qui profitent aux zones les moins accessibles. En reprenant la logique de la théorie du surplus nous pouvons ainsi comparer deux types de critère: l'un proposé par le calcul économique standard, dont l'objectif est l'efficacité, l'autre, correspondant à la vision rawlsienne, le critère du maximin, conduisant lui à retenir le projet qui maximise la variation du surplus là où l'accessibilité est la plus faible.

Cependant, une critique est fréquemment formulée contre ce « critère du maximin », dans la mesure où il peut aboutir à des conséquences que l'on peut juger difficilement admissibles. Par exemple, une action politique qui laisserait inchangée la situation de la zone la plus défavorisée mais améliorerait plusieurs autres zones très démunies serait elle nécessairement moins favorable, au sens d'une plus grande équité, qu'une action politique bénéficiant à la seule zone la plus défavorisée ?

Une deuxième critique peut être faite à ce principe du maximim dès lors qu'il est appliqué à des inégalités spatiales. Le risque d'être un jour parmi les plus pauvres, qui fonde l'approche de RAWLS, est-il de même nature, en termes d'incertitude ou de probabilité, que celui d'avoir à habiter dans la zone la moins bien desservie ?

Enfin, on pourrait faire une dernière critique en reprenant PIKETTY (1994) visant à se demander comment la collectivité parvient à une connaissance objective de l'identité des membres les plus défavorisés de la société ou, pour ce qui nous concerne, de la zone la plus démunie en termes d'accessibilité : est-elle en Auvergne, dans les Hautes-Alpes ou quelque part en Corse ?

\subsubsection{L'intégration d'un facteur d'équité dans l'expression du surplus}

Au delà de ces théories, l'équité spatiale trouve plusieurs définitions (LLUGANY, 2000). Selon BIDET, l'équité se définit comme «l'égalité démocratique, qui tend à la maximisation de la position des plus favorisés » (BIDET, 1995). C’est ainsi accroître la présence médicale auprès des populations lésées géographiquement. Pour BEGUIN (1989), « au niveau d'un seul agent, efficacité et équité se confondent, l'efficacité consiste à optimiser l'objectif commun, donc moyen; l'équité consiste à accorder de l'attention à certains membres de la collectivité, c'est-à-dire à modifier les localisations dans un sens qui leur soit favorable. Dès lors, le système des localisations n'est plus l'optimum collectivement efficace. L'équité s'oppose à l'efficacité, la collectivité abandonne une partie de son efficacité globale pour améliorer le sort d'une partie de ses membres : c'est l'expression de la solidarité ». 
L’expression néo-utilitariste de la fonction d'utilité collective peut être reprise afin de dépasser ces critiques et d'aborder de façon formalisée le conflit entre équité et efficacité dans la problématique de l'évaluation des politiques de transports. Pour ce faire, nous proposons de pondérer la fonction de surplus des usagers du calcul économique par un indicateur d'accessibilité affecté d'un exposant $-\alpha$. La formule [2] devient alors :

$$
\Delta \mathrm{S}=\Delta \mathrm{R}+\Delta \mathrm{C}+\sum_{\mathrm{i}} \Delta \mathrm{S}_{\mathrm{i}} \mathrm{A}_{\mathrm{i}}^{-\alpha}
$$

Cette fonction, inspirée de l'approche néo-utilitariste et des travaux de THISSE ${ }^{12}$, peut être analysée comme l'expression d'un compromis entre le critère d'efficacité et celui d'équité. En effet, lorsque $\alpha=0$, on retrouve l'expression ${ }^{13}$ de la fonction utilitariste du surplus du calcul économique, et pour $\alpha>0$, on obtient une fonction intégrant une norme d'équité. (elle se rapproche du critère du maximin lorsque $\alpha$ est très grand). $\alpha$ s'interprète comme une mesure de l'aversion contre l'iniquité spatiale qui caractérise les préférences du décideur, et sa valeur traduit un compromis entre les deux critères d'efficacité et d'équité. Ainsi, en fonction des valeurs de $\alpha$ fixées, la maximisation de cette fonction peut aboutir à sélectionner des projets de transports dont les orientations sont de nature très différente.

Nous pouvons considérer que cette fonction, autorise une formalisation des travaux effectués par BLOY et alii (1976) sur la présentation de la contradiction entre efficacité et équité. Les auteurs proposent, dans une évaluation en termes de surplus global, de ventiler le surplus des usagers selon les zones de l'espace considéré comme nous l'avons proposé cidessus. Les iniquités spatiales de la situation initiale sont représentées par un vecteur d'indicateurs d'accessibilité. Pour analyser le caractère redistributif des projets, les auteurs proposent de pondérer chaque zone par sa population et de calculer une droite de régression à partir du nuage de points défini par la distribution $\left\{\mathrm{A}_{\mathrm{i}}, \Delta \mathrm{S}_{\mathrm{i}}, \mathrm{h}_{\mathrm{i}}\right\}$, avec $\mathrm{A}_{\mathrm{i}}$ l'indicateur d'accessibilité de la zone $\mathrm{i}, \Delta \mathrm{S}_{\mathrm{i}}$ la variation du surplus bénéficiant aux habitants de la zone $\mathrm{i}$, et $h_{i}$ le poids démographique de celle-ci. En dépit de la simplification, peut-être excessive, que constitue cette représentation linéarisée, le faisceau, formé par l'ensemble des droites représentatives des politiques alternatives, fournit deux informations susceptibles d'éclairer le décideur entre le choix d'une politique efficace et le choix d'une politique redistributive :

- La « hauteur» de chacune des droites (au sens de l’ordonnée du point moyen) représente la valeur totale de la variation de surplus inhérente à la stratégie, c'està-dire, le critère traditionnel du calcul économique ;

- La pente de chacune des droites indique le caractère correctif de la stratégie, (plus la droite a une pente négative, plus la stratégie a des effets redistributifs et inversement).

Notons que les positions relatives des droites qui représentent des solutions alternatives sur la figure ne résultent pas d'un dessin ad hoc destiné à justifier le raisonnement, mais bien de la contradiction que nous avons formalisée et démontrée au paragraphe 2.2. 
$\underline{\text { Figure } 2}$ - Représentation graphique des arbitrages entre efficacité et équité spatiale

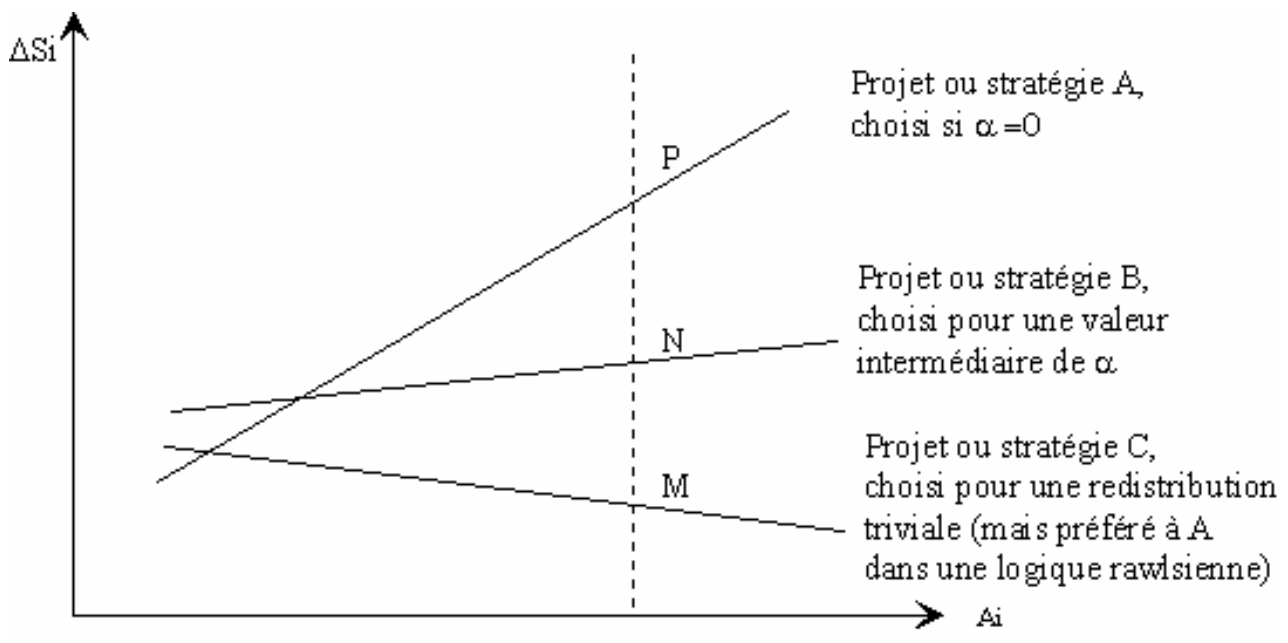

D’après cette représentation, l'arbitrage entre efficacité et équité revient à mettre en balance d'une part la « hauteur » des droites et, d'autre part, leur pente. Ainsi, si l'on compare les trois variantes représentées, le projet $\mathrm{A}$ est le meilleur en termes d'efficacité, en dépit d'une pente fortement positive. A contrario, le projet $\mathrm{C}$ induit une correction des inégalités spatiales (pente décroissante) au détriment de l'efficacité économique. En ce qui concerne la stratégie $\mathrm{B}$, la droite présente une pente faiblement positive et une hauteur à mi-chemin entre les projets $\mathrm{A}$ et $\mathrm{C}$. Dans la comparaison qui peut être faite entre les trois options, notons que les arbitrages renvoient bien à des conceptions très normatives de la redistribution :

- l'option C n'est préférable aux deux autres que dans une conception triviale de la redistribution (les moins favorisés reçoivent plus que les plus favorisés) qui lui sera préférée. Elle est cependant préférable à l'option A pour un $\alpha$ très élevé (option rawlsienne),

- l'option $B$, bien qu'elle favorise les mieux pourvus, sera préférée à $C$ pour toute valeur de $\alpha$. Elle sera préférée à A pour un $\alpha$ intermédiaire et, a fortiori pour un $\alpha$ élevé.

- L’option A sera préférée aux deux autres avec une valeur faible de $\alpha$.

Cette présentation offre l'avantage de donner une mesure du coût de la redistribution (la distance PM par exemple). Un usage combiné de ce type de représentation et de calculs utilisant la pondération de l'équation [23] a ainsi le mérite de représenter tout à la fois le classement induit par une valeur de $\alpha$ (par exemple entre les projets $\mathrm{A}, \mathrm{B}$ ou $\mathrm{C}$ ) et la perte de surplus global $^{14}$ que peut impliquer une option redistributive. Cependant, il est certain que cette approche reste fragile en raison de la part d'arbitraire liée à la fixation de la valeur de $\alpha$. Celle-ci ne peut être réduite que par la pratique, qui reste à engager, de telles évolutions qui procureraient des valeurs limites de $\alpha$ pour lesquelles des classements de projets concrets seraient susceptibles de basculer. 


\section{QUELLE CONCLUSION POUR LA POLITIQUE DES TRANSPORTS ET DE L'AMENAGEMENT}

Nous avons montré qu'il existait une certaine contradiction entre efficacité et équité spatiale, et nous avons tenté de la formaliser. Face à cette contradiction, le calcul économique fait un arbitrage clair mais peu satisfaisant puisqu'il conduit à maximiser la seule rentabilité d'un projet. Les propositions que nous avons développées suggèrent que cet arbitrage peut être moins brutal mais qu'il ne peut être du ressort du seul économiste car il relève, en somme, d'une ambition pour le territoire. Il semble, cependant, que les instruments d'analyse proposés permettent d'aller plus loin que cette séparation des rôles de l'économiste et de l'aménageur, non point sous la forme d'une préconisation économique qui se réclamerait d'un principe d'optimalité, mais dans la perspective d'un meilleur éclairage d'options que l'on sait sous-optimales mais qui peuvent participer à une réduction des déséquilibres territoriaux.

En jouant avec ce que permettent d'exprimer les indicateurs d'accessibilité et ce que permet de mesurer le calcul économique, notamment la perte de surplus qui peut résulter du choix redistributif, nous proposons, en somme, que puisse être mise en balance l'ambition territoriale et son coût collectif. Cela peut suggérer un dispositif de décision et de financement plus transparent : la maximisation du surplus global de productivité incomberait toujours à la politique de transport et à ses budgets; la redistribution territoriale incomberait à l'aménagement du territoire et à ses budgets. Un choix sous-optimal du point de vue de la politique des transports reste possible et serait d'autant plus légitime que son coût collectif n’en serait plus caché et qu’il serait couvert par une affectation délibérée de ressources.

Pour autant, cela ne fait que déplacer les limites du calcul économique dans un sens qu'évoquait déjà un rapport du Commissariat au Plan. "Le calcul économique s'arrête là où les grands choix structurants commencent [...] les arbitrages d'aménagement économiques peuvent être renseignés par l'économiste, mais les choix sont opérés par le politique. C'est ce dernier qui arbitre entre efficacité et équité, entre deux parts de l'effort national : celle que l'on consacre à renforcer les atouts des régions et des agglomérations bien placées par rapport aux grands courants économiques internationaux; celle qui est affectée à améliorer l'accessibilité des régions moins avantagées [...]. Les calculs économiques permettent de chiffrer le prix des solidarités, de mesurer les investissements plus rentables éludés par un choix, mais seule une analyse politique et des choix stratégiques permettent de trancher ${ }^{15}$. 


\section{BIBLIOGRAPHIE}

AFFICHARD J., de FOUCOULD J.B. eds (1992), Pluralisme et équité, Paris, Editions Esprit. AYDALOT Ph. (1985). Economie régionale et urbaine. Economica, Paris.

BAUMSTARK L., BONNAFOUS A. (2000). «La relecture théorique de Jules Dupuit par Maurice Allais face à la question du service public ", in Les traditions économiques françaises, 1848-1939, CNRS Ed, avril 2000, pp. 397-412.

BEGUIN H., ZOLLER H. (1992). Aide à la décision, l'évaluation des projets d'aménagement, Paris, Bibliothèque de Sciences Régionales, Economica, , 273 p.

BEN AKIVA M., LERMAN S. (1979). « Disagregate travel and mobility choice models and measures of accessibility », in HENSHER D.A., STOPHER P.R., Behavioural travel modelling, Groom Helm London, pp. 698-710.

BEN AKIVA M., LERMAN S. (1985). Discrete choice analysis - Theory and application to travel demand, The MIT Press, Cambridge.

BLANCHARD O., CRIQUI P., TROMETTER M., VIGUIER L. (2000), «Au-delà de Kyoto: enjeux d'équité et d'efficacité dans la négociation sur le changement climatique », Economie et Prévision, n¹43-144, pp. 15-35.

BONNAFOUS A. (1992) «Calcul économique et objectifs d'aménagement du territoire ». in 6th Conférence Mondiale sur le recherche dans les transports. Volume I, Espace, développement et globalisation, Lyon.

BOYER R. (1992), «Justice sociale et performa,nces économiques: de la synergie au conflit ? » in AFFICHARD J., de FOUCOULD J.B. eds (1995), Pluralisme et équité, Paris, Editions Esprit, pp. 33-71.

BLOY E., BONNAFOUS A., CUSSET J.M., GERARDIN B. (1976). Evaluer la politique des transports. Economica, Paris, PUL, Lyon.

CAZENAZE Ph., MORRISON Ch. (1978). Justice et redistribution. Economica, Série Sciences Economiques, Paris.

CRECY R. (1979). «Quelques réflexions sur l’accessibilité ». Les cahiers scientifiques de la revue Transports, 4ème trimestre.

ERLANDER S., STEWART N.F.(1990). The gravity model in transportation analysis Theory and extensions. VSP, Utrech.

FUSTIER B. (1988). «Les interactions spatiales ». in PONSARD C. (sous la direction de). Analyse économique spatiale, PUF, Paris.

GUENGANT A. (1993). "Equité, efficacité et égalisation fiscale territoriale ». Revue Economique, $\mathrm{N}^{\circ} 4$, pp. 835-848. 
HANSEN W.G. (1959). « How accessibility shapes land use », American Institute of planners journal, 25, 73-76.

KOENIG G. (1979). «A propos de : «Quelques réflexions sur la notion d'accessibilité ». Les cahiers scientifiques de la revue Transports, 4ème trimestre.

KOENIG G. (1974). «Théorie économique de l'accessibilité urbaine ». Revue Economique, $\mathrm{N}^{\circ} 2$.

LESOURNE J. (1972). Le calcul économique. Dunod, Paris.

LLUGANY J. (2000). « De la commune au quartier : la médecine de ville libérale de premier recours en 1990 dans les Pyréennées-Orientales », Les Actes du FIG 2000, Géographie et Santé.

MONNIER J.-M. ed (1999). Dynamiques économiques de l'équité, Paris, Economica, Collection Grands débats.

MORRIS J.M., DUMBLE P.L., WIGAN M.R. (1978). Accessibility indicators for transport planning, in Transportation Research - A, pp. 91 - 109.

NEUBERGER H. (1971). " User benefit in the evaluation of transport and land use plans ». Journal of transport economics and policy. Janvier.

PIKETTY T. (1994). Introduction à la théorie de la redistribution des richesses. Collection « Approfondissement de la connaissance économique », Economica, Paris, 116 p.

PIKETTY T. (1997). Economie des inégalités, Editions La découverte et Syros, Paris.

POULIT J. (1974). Urbanisme et transport : les critères d'accessibilité et de développement urbain. SETRA, Division urbaine, Ministère de l'équipement, Paris, 55 p.

RAWLS (1987). Théorie de la justice. Edition du Seuil, Paris.

RICOEUR P. (1992). «Unicité ou pluralité des principes de justice » in AFFICHARD J., de FOUCOULD J.B. eds (1992), Pluralisme et équité, Paris, Editions Esprit.

THISSE J.F. (1994). «L'équité spatiale ». in AURAY J.P., BAILLY A., DERYCKE P.H., HURIOT J.M., (sous la direction de). Encyclopédie d'Economie Spatiale-Concepts, comportements, organisations. Bibliothèque de Science Régionale, Economica, Paris, pp. 225-232.

WACHS M., KOENIG G. (1979). « Behavioural modelling, accessibility, mobility and travel need ». in HENSHER D.A., STOPHER P.R. (Eds), Behavioural travel modelling, Groom Helm London.

WILLIAMS H.C.W.L., SENIOR M.L. (1978). "Accessibility, spatial interaction and the spatial benefit of land use-transportation plans ». in KARLQVIST A., LUNDQVIST L., 
SNICKARS F., WEIBULL J.W. (Eds). Spatial Interaction Theory and Planning Models. North Holland, 253-287.

WILSON A. G. (1970). Entropy in urban and regional modelling. Pion, London.

WOLFELSPERGER A. (1995). Economie publique. Collection Thémis Economie, P.U.F., Paris.

${ }^{1}$ Ce qui revient à ventiler par zone la part de la valeur actualisée nette (V.A.N) du projet ou de la politique considérée qui résulte des variations de surplus des usagers, les autres parts correspondant à des recettes ou dépenses de la collectivité que l’on peut considérer comme indivisibles (Cf. éq. [2] infra).

${ }^{2}$ BLOY, BONNAFOUS et alii (1976).

${ }^{3}$ WILLIAMS et SENIOR (1978) ont montré que cette mesure, élaborée sous des conditions de changements marginaux, est une bonne approximation de la mesure du surplus du consommateur de HOTELLING (1938) qui lui même reprend, enrichi de sa formalisation mathématique, le concept de surplus de l'usager de Jules DUPUIT (BAUMSTARK, BONNAFOUS, 2000). Ajoutons que LESOURNE (1972) a généralisé ce résultat aux changements qui induisent des différences finies.

${ }^{4}$ GOULD (1969, in MORRIS, et alii, 1978) souligne le caractère glissant de l'accessibilité, en rappelant qu'il s'agit d'un terme que chacun utilise communément jusqu'à ce qu'il soit confronté au problème de définition et de mesure.

${ }^{5}$ Le passage des formules [17] à [18] peut paraître surprenant. En effet, l'expression [18] ne s'obtient pas en réalisant la somme sur $\mathrm{j}$ des $\mathrm{S}_{\mathrm{ij}}$, mais par la transposition de l'expression de la satisfaction ressentie par l'usager pour une liaison donnée, à celle ressentie par l'usager pour l'ensemble des liaisons issues de i. Pour une liaison ij, c'est le log des emplois à destination pondéré par la fonction de résistance qui est considéré, et pour l'ensemble des liaisons issues de i, il s'agit donc l'ensemble des emplois offerts en destination (leur somme) pondéré par leurs fonctions de résistance.

${ }^{6}$ Formulation reprise de : WACHS M., KOENIG G. (1979).

${ }^{7}$ On peut souligner que cette approche dite « néo-utilitariste " s'intéressant à définir des fonctions d'utilité collective spécifique ne constitue pas l'unique alternative au modèle standard. On peut également intégrer indirectement dans la théorie utilitariste un souci de justice par l'intermédiaire des préférences que les individus peuvent avoir eux-mêmes à cet égard. Autrement dit, au lieu de s'interroger sur la forme de l'utilité collective, on peut redéfinir les fonctions d'utilités individuelles en les considérant comme interdépendantes. L’individu n’est alors plus considéré comme parfaitement égoïste, mais prend en considération le niveau d'utilité des autres.

${ }^{8}$ D'un point de vue néo-utilitariste se prononcer sur la justice c'est exprimer une préférence de nature éthique sur les états de l'économie définis exclusivement par les niveaux respectifs d'utilité atteints par les différents individus concernés, toute théorie de la justice répondant à une exigence minimale de rationalité (transitivité) correspond à un "préordre sur les assortiments d'utilités individuelles » de la même manière que les préférences des individus peuvent être représentées par un préordre sur les assortiments de biens. Selon une certaine tradition de la philosophie morale, on peut l'attribuer à un être fictif qualifié « d'observateur idéal ». A la fois rationnel, parfaitement informé, impartial et bienveillant, l'observateur idéal étend également sa sympathie à l'ensemble des membres de la société. Ce préordre est total. C'est ce qui permet d'y avoir recours pour sortir de l'indétermination résultant de l'usage du seul critère de Pareto. On voit ainsi que, sous la condition de continuité nécessaire pour pouvoir faire correspondre une fonction à ce préordre, toute théorie de la justice de type utilitariste peut être synthétisée sous la forme d'une fonction telle qu'à chaque ensemble de valeurs prises par les utilités individuelles corresponde une valeur d'un indicateur qui «mesure » la justice de chacune de ces répartitions. (WOLFELSPERGER, 1995). 
${ }^{9}$ WOLFELSPERGER A. (1995). Economie publique. Collection Thémis Economie, P.U.F, Paris.

10 RAWLS, A theory of justice. The belknap press of Harvard University Press. 1971. Pour la traduction française, RAWLS, Théorie de la justice, Edition du Seuil, 1987.

${ }^{11}$ L'hypothèse d'égoïsme a pour avantage de ne pas fonder le principe de justice de manière ad hoc et fragile sur une quelconque sensibilité à l'inégalité qui traduirait une préoccupation altruiste pour le bien être des autres.

${ }^{12}$ C'est dans une logique semblable que THISSE applique au problème weberien (localisation d'un service public en fonction des distances aux usagers) un coefficient $\alpha$ exprimant les préférences du décideur.

${ }^{13}$ Cf. l'équation [4]

${ }^{14}$ En toute rigueur, les distances PN ou PM devraient être corrigées des éléments autres que les surplus d'usagers mais qui composent également la valeur actualisée nette de chaque projet.

${ }^{15}$ Commissariat général au Plan. Transports : le prix d’une stratégie. Tome 1 : éléments de réflexion et recommandation. Tarification et financement. Atelier présidé par Alain BONNAFOUS. La Documentation Française, Paris, 1995. 